\title{
Article
}

\section{Trait emotional intelligence and attentional bias for positive emotion: An eye tracking study}

Lea, Rosanna G., Qualter, Pamela, Davis, Sarah K., Pérez-González, Juan-Carlos and Bangee, Munirah

Available at http://clok.uclan.ac.uk/21890/

Lea, Rosanna G., Qualter, Pamela, Davis, Sarah K., Pérez-González, JuanCarlos and Bangee, Munirah ORCID: 0000-0001-8548-6692 (2018) Trait emotional intelligence and attentional bias for positive emotion: An eye tracking study. Personality and Individual Differences, 128 . pp. 88-93. ISSN 0191-8869

It is advisable to refer to the publisher's version if you intend to cite from the work. http://dx.doi.org/10.1016/j.paid.2018.02.017

For more information about UCLan's research in this area go to http://www.uclan.ac.uk/researchgroups/ and search for <name of research Group>.

For information about Research generally at UCLan please go to http://www.uclan.ac.uk/research/

All outputs in CLoK are protected by Intellectual Property Rights law, including Copyright law. Copyright, IPR and Moral Rights for the works on this site are retained by the individual authors and/or other copyright owners. Terms and conditions for use of this material are defined in the policies page.

\section{CLoK}

Central Lancashire online Knowledge www.clok.uclan.ac.uk

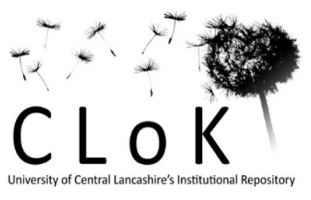




\begin{abstract}
Emotional intelligence (EI) may promote wellbeing through facilitation of adaptive attentional processing patterns. In the current study, a total of 54 adults (43 females, mean age $=25$ years, $\mathrm{SD}=10$ years $)$ completed a Trait Emotional Intelligence (TEI) scale and took part in three eye-tracking tasks, where they viewed (1) faces with different emotions (happy, angry, fearful, neutral), (2) 16-face crowds with varying ratios of happy to angry faces, and (3) 4 visual scenes (physical threat, social threat, positive social, neutral). Findings showed that higher TEI was associated with more attention to positive emotional stimuli (happy faces, positive social scenes), relative to negative and neutral stimuli. An attentional preference for positive rather than negative emotional stimuli may be one way that TEI affords protection from stressors to promote mental health.
\end{abstract}

Trait emotional intelligence and attentional bias for positive emotion: An eye tracking study

\title{
1. Introduction
}

Emotional intelligence (EI) captures individual differences in how people perceive, regulate, use, and understand their own emotions and the emotions of others (Nelis, Quoidbach, Mikolajczak, \& Hansenne, 2009). EI can be conceptualised in two ways: (1) trait EI (TEI), referring to a constellation of emotional perceptions assessed through questionnaires and rating scales (Petrides, Pita, \& Kokkinaki, 2007); and (2) ability EI (AEI), which concerns emotion-related skills and competencies, measured as 'maximum performance', akin to cognitive ability (Mayer, Roberts, \& Barsade, 2008). EI is seen as a core individual difference, relating to positive behavioural outcomes across multiple areas of 
functioning, including relationships, educational achievement, and occupational success (Brackett, Rivers, \& Salovey, 2011; Petrides et al., 2016). In particular, EI is a robust predictor of mental health (Martins, Ramalho, \& Morin, 2010).

Researchers tend to study the 'static' structure of EI and its descriptive associations with life outcomes rather than its mechanisms of action. In order to substantiate claims of EI as an agent of improved wellbeing, we need to better understand how EI works, and specifically how EI relates to key cognitive processes, such as attention (Fiori, 2009; Gutiérrez-Cobo, Cabello, \& Fernández-Berrocal, 2016). Since TEI maps onto temperament and personality-related factors (e.g. optimism) that have been shown to influence attentional processing of emotion (Kress \& Aue, 2017), it is expected that TEI would also have a role in these processes. Although investigating the input of AEI is also important, there is a pressing need to verify the nomological net of TEI as a non-cognitive, lower order personality trait (Petrides et al., 2016). The focus for the current study is therefore on TEI, rather than AEI.

\subsection{Attentional Bias to Emotion: A Role for Trait Emotional Intelligence?}

The allocation of attentional resources to emotional stimuli may be one way that EI buffers the effects of stress, ultimately, promoting wellbeing (Davis, 2017; Matthews et al., 2015). Fiori (2009) postulates that EI should facilitate attention to emotional information. However, if TEI is adaptive, according to extant literature on adaptive attentional processing (Mogg \& Bradley, 1998; Weierich, Treat, \& Hollingworth, 2008, Yiend, 2010), higher levels should relate to reduced attention towards threatening/negative emotional material in nonstressful conditions, and vice versa in stressful conditions. Such an attentional profile would also be consistent with findings indicating an attention and memory bias toward positive emotional content in optimistic people (Kress \& Aue, 2017), given measures of TEI tap that trait. 
A systematic review assessed the relationship between EI and cognition (e.g. attention, memory, decision-making processes) through laboratory tasks (Gutiérrez-Cobo et al., 2016). Of the 26 studies identified, only four used attention for the primary cognitive focus (3 TEI; 1 AEI), making drawing conclusions about attentional processes and EI challenging. Mikolajczak, Roy, Verstrynge and Luminet (2009) identified significant effects in a word dot probe task: those with high TEI (though only trait self-control) allocated more attentional resources towards emotional words (versus neutral words) under stressful conditions. In a word-based emotional Stroop task, Coffey et al. (2003) found a positive relationship between TEI (attention to emotion subscale) and attention towards emotional (relative to non-emotional) words, whereas Fisher et al. (2010) identified a negative relationship between TEI and attention to negative stimuli. However, Matthews et al. (2015) found no association between TEI and attentional processing of either emotional (faces) or neutral (nuts) stimuli. Overall, findings linking EI to attentional processing of emotional stimuli are mixed.

A limitation of the above studies concerns ecological validity, namely the use of words, or isolated faces, as a stimuli source, rather than salient emotional images (Bar-Haim et al., 2007). Because emotional faces are rarely presented in isolation in everyday life, stimuli such as the 'face in the crowd' paradigm, or emotional scenes, are more socially relevant (Pinkham, Griffin, Baron, Sasson, \& Gur, 2010; Yiend, 2010). It is also worth noting that studies only examined the link between TEI and attention towards emotional stimuli in general (versus neutral), without testing whether the relationship is contingent on emotion type (i.e. positive, negative). Furthermore, in the field of attentional processing, eye-tracking techniques provide a more rigorous paradigm whereby attention can be directly and continuously measured, compared to behavioural measures such as reaction time data (Waechter, Nelson, Wright, Hyatt, \& Oakman, 2014). Only one study has examined the 
relationship between EI and attention to emotion via eye movements (Davis, in press). In that dot-probe study, participants’ attentional fixation towards happy, sad, angry, and neutral faces were captured in either stressful or non-stressful conditions. A complex myriad of effects were found for TEI and AEI, with findings challenging the notion of TEI as an adaptive construct because it was associated with hypervigilance towards angry and sad faces.

\subsection{The Present Study}

The current study is the first to use a range of ecologically valid emotional stimuli ('face in the crowd' paradigm; emotional scenes) to probe the influence of TEI on attentional processing using eye-tracking technology. Based on theory that threat avoidance in nonstressful situations confers adaptive processing (Mogg \& Bradley, 1998), and the assumption that high TEI individuals might have similar attentional bias toward positive stimuli to those high in trait Optimism (e.g., Kress \& Aue, 2017), two hypotheses were tested. H1 predicted that there would be a negative association between TEI and fixation time for negative emotional material, relative to positive and neutral material; H2 predicted that finding would generalise to different stimuli types (i.e. faces, crowds, scenes). An implicit assumption prevails in the literature that higher EI is always adaptive, but that may not be the case. Both Davis and Nichols (2016) and Qualter, Whiteley, Hutchinson, and Pope (2007) discuss the idea of an EI threshold, where there is an optimum level before effects plateau (or become negative). Hence, the present study included quadratic analyses to capture any non-linear effects of EI on attention processing.

\section{Method}

\subsection{Participants and Procedure}


54 UK adults (43 females; 11 males) with a mean age of 25 years ( $S D=10$ years) were recruited from staff and student cohorts at a University in the North West of England, UK. Ethical approval was obtained from the University Ethics Committee. The same sample participated in all three experimental tasks. Upon arrival to the experimental room, informed consent was obtained, and participants completed the Trait Emotional Intelligence Questionnaire - Short Form (Petrides, 2009). Participants took part in three passive pictureviewing tasks outlined below (with presentation order counterbalanced) where eye movements were monitored using eye-tracking technology. The eye-tracker was calibrated for each participant. Total testing time was approximately 45 minutes.

\subsection{Materials and Measures}

\subsubsection{Emotional Intelligence}

Emotional intelligence was measured using the TEIQue-SF (Petrides, 2009), where individuals indicate their level of agreement/disagreement with a set of 30 brief statements using a 7-point Likert scale ('Completely disagree’ [1] to ‘Completely agree’ [7]). Global scores and scores on four emotional self-perception factors: 'wellbeing' (e.g. 'On the whole, I’m pleased with my life’), 'self-control’ (e.g. 'I tend to change my mind frequently'), 'emotionality’ (e.g. 'I often pause and think about my feelings') and 'sociability' (e.g. 'I can deal effectively with people') were calculated. The TEIQue-SF has a robust factor structure, excellent reliability ( $\alpha=.88-.92)$, and good item discrimination (Cooper \& Petrides, 2010). The present study achieved adequate reliability scores ranging from $\alpha=.67$ (emotionality) to $\alpha=.80$ (sociability; global score).

\subsubsection{Eye-Tracking System}

A fixed Eyelink II eye-tracker, with monocular recording at 500Hz (SR Research, US), was used to track eye movements on an individual basis for each task. Attention was 
conceptualised in terms of eye fixations on specified areas of interest (i.e. images of faces, scenes etc.), with this information captured using EyeLink Data Viewer. An eye fixation was recorded when the participant had a saccade in any of the areas of interest that were previously coded in the software. A fixation occurrence was determined according to a standard logarithm of at least $100 \mathrm{~ms}$ in a given radius of 0.5 degrees of visual angle.

\subsubsection{Experimental tasks}

\subsubsection{Task 1: EI and Attentional Bias to Emotional Faces}

The first task examined the relationship between TEI and attentional bias towards emotional faces. Emotional facial stimuli were selected from the Karolinska directed emotional faces database (KDEF; Lundqvist, Flykt \& Öhman, 1998). All KDEF images have been validated, with excellent (88\%) test-retest reliability (Goeleven, De Raedt, Leyman, \& Verschuere, 2008). In a 2x2 matrix, four emotional expressions (happy, angry, afraid, neutral) of the same person were presented simultaneously. Happy faces reflected positive emotion, angry and fearful faces represented threatening cues, and neutral faces were non-emotive control stimuli. Each matrix was randomised such that any of the four expressions could present in one of four locations (i.e. top left, top right, bottom left, bottom right), with an equal number of male and female faces. Participants viewed all 24 picture slides (each containing one 2x2 matrix) in a random order. Each slide was viewed for 8 seconds, followed by a 5 second blank screen, and a central fixation point (which participants focussed on between trials).

\subsubsection{Task 2: EI and Attentional Bias to Emotional Faces in a Crowd}

The second task examined the relationship between TEI and attentional bias towards emotional faces in a crowd. Adjusting the ratio of happy to angry faces is employed by studies (e.g. Lange et al., 2011) to test sensitivity to social threat. Photographs of 16 male 
individuals with happy and angry facial expressions were selected from the KDEF (Lundqvist, Flykt \& Öhman, 1998), resized to 170 x 113 pixels, and used to produce 'crowd' matrices composed of 16 different faces $(4 \times 4)$. Based on a previous study (Lange et al., 2011), seven happy-angry crowd types were created by increasing the ratio of happy to angry faces within each crowd e.g. 14:2 (14 happy, 2 angry), 12:4, 10:6, 8:8, 6:10, 4:12, 2:14. Participants viewed 21 slides containing one crowd on each (3 slides for each of the 7 crowd types) in a randomised order. Each slide was viewed for 8 seconds, followed by a 5 second blank screen, and a central fixation point. Male faces were used due to faster processing speeds for male faces within this paradigm (Lange et al., 2011).

\subsubsection{Task 3: EI and Attentional Bias to Emotional Scenes}

The final task examined the relationship between TEI and attentional bias towards emotional scenes. Pictures of scenes were selected from the International Affective Picture System (IAPS; Lang, Bradley \& Cuthbert, 2008). In a 2x2 matrix, four visual scene types were presented simultaneously: physical threat (violence, aggression), social threat (rejection, lone individuals), social positive (social interaction, social relationships), and neutral (nature). Each matrix was randomised such that any of the four images could present in any of the four locations (i.e. top left, top right, bottom left, bottom right). All 24 picture slides (each containing one 2x2 matrix) were shown to the participant in a random order. Each slide was viewed for 8 seconds, followed by a 5 second blank screen and a central fixation point, which participants were asked to focus on between trials.

\subsection{Data Analyses}

In Task 1 and Task 3, three time blocks were used to capture attentional processing over time: ‘very early’ (0-500ms), ‘early’ (500-4000ms) and 'late’ (4000-8000ms), aligning the present study with other eye-tracking studies as far as possible (Buckner, Maner, \& 
Schmidt, 2010; Jang, Kim, Kim, Lee, \& Choi, 2016). Time blocks were not used for Task 2, following recommendations regarding the 'face in the crowd' paradigm (Armstrong \& Olatunji, 2012). For Task 1, the mean proportion of time spent fixating on each facial expression, relative to total fixation time, was computed per time block across the 24 slides. For Task 2, the mean proportion of time spent fixating each emotion type (happy; sad) faces relative to total fixation time for each crowd type (e.g. 10:6; 8:8), was computed. For Task 3, the mean proportion of time spent fixating on each scene type, relative to total fixation time, was computed per time block across the 24 slides. Linear and quadratic (curvilinear) regressions were conducted for all tasks to test for relationships between TEI and attentional biases. Chi square analyses were used to assess whether initial area of fixation varied according to level of TEI (high versus low). All analyses were repeated with global and TEI factor scores to allow a fine-grained analysis of the role of constituent factors in attentional processes (e.g., Matthews et al., 2015).

\section{Results}

\subsection{Task 1: EI and Attentional Bias to Emotional Faces}

Linear and curvilinear regressions were performed, with TEI (i.e. TEIQue-SF global or factor score) as the predictor variable, and mean proportion of fixation time for each emotion type (happy, angry, afraid, neutral) for each time block (very early, early, late), as criterion variables. No linear or quadratic associations were identified for TEI (nor its component factors) and fixation time for afraid (L: $\beta s \leq-.05, p s>.05$; Q: $\beta s \leq-.66, p s>.05$ ), angry (L: $\beta \mathrm{s} \leq .02$, ps >.05; Q: $\beta \mathrm{s} \leq 1.00, p s>.05)$, happy $(\mathrm{L}: \beta \mathrm{s} \leq .15, p s>.05 ; \mathrm{Q}: \beta \mathrm{s} \leq-.79$, ps $>.05$ ) or neutral ( $\mathrm{L}: \beta \mathrm{s} \leq .08$, ps $>.05$; $\mathrm{Q}: \beta \mathrm{s} \leq .02$, $p s>.05$ ) faces, regardless of time block. Results indicate that TEI was not associated with attention towards any particularly emotional facial expression. 
The emotion of first fixation did not vary between those classified as high or low in TEI, $\chi^{2}(3, \mathrm{~N}=54)=4.56, \mathrm{p}=.207$ : individuals, first, looked at the afraid face, regardless of TEI level.

\subsection{Task 2: EI and Attention Bias to Emotional Faces in a Crowd}

Linear and curvilinear regressions were performed, with TEI (i.e. TEIQue-SF global or factor score) as the predictor variable, and mean proportion of fixation time for each emotion type (happy, angry) for each crowd type, as criterion variables. No linear or quadratic associations were identified for global TEI and fixation time for angry faces in any of the seven crowd types: 14 happy and 2 angry $(\mathrm{L}: \beta=-.02, p=.95 ; \mathrm{Q}: \beta=-6.03, p=.95)$, 12 happy and 4 angry (L: $\beta=.02, p>.87 ; \mathrm{Q}: \beta=2.31, p>.43), 10$ happy and 6 angry (L: $\beta=$ -.79, $p=.58 ; \mathrm{Q}: \beta=2.25, p=.39), 8$ happy and 8 angry $(\mathrm{L}: \beta=.16, p=.25 ; \mathrm{Q}: \beta=-1.87, p$ $=.30), 6$ happy and 10 angry (L: $\beta=-.26, p=.068 ; \mathrm{Q}: \beta=-.48, p=.19), 4$ happy and 12 angry (L: $\beta=.00, p=.98 ; \mathrm{Q}: \beta=-.21, p=.99)$, or 2 happy and 14 angry $(\mathrm{L}: \beta=.01, p=.95$; Q: $\beta=-.20, p=.99)$. In addition, no associations were found between any TEI factors and fixation times on angry faces in any crowd type.

A significant linear, but not quadratic, relationship was found between global TEI and fixation time for happy faces in the crowd with 6 happy and 10 angry faces $(\mathrm{F}(1,52)=8.84, p$ $=.019$, adjusted $\mathrm{R}^{2}=.085$; see Figure 1 ). Specifically, global TEI score had a positive relationship with happy face fixating time $(\beta=.32)$. Significant positive linear, but not curvilinear, relationships were also identified between happy face fixation and some TEI factors. These were present for (1) emotionality: 6 happy and 10 angry $(\beta=.32, p=.019)$, and 2 happy and 14 angry ( $\beta=.29, p=.039)$; (2) self-control: 14 happy and 2 angry $(\beta=.39$, $p=.004), 10$ happy and 6 angry $(\beta=.30, p=.032), 6$ happy and 10 angry $(\beta=.41, p=.002)$, and 2 happy and 14 angry $(\beta=.33, p=.016)$; (3) wellbeing: 6 happy and 10 angry $(\beta=.43, p$ 
$=.001)$. In sum, individuals with higher TEI showed a visual preference towards happy faces in a group context, particularly if the crowd was formed mostly of angry faces.

\subsection{Task 3: EI and Attentional Bias to Emotional Scenes}

Linear and curvilinear regressions were performed, with TEI (i.e. TEIQue-SF global or factor scores) as the predictor variable, and mean proportion of fixation time for each emotion type (physical threat, social threat, social positive, neutral) for each time block (very early, early, late) as criterion variables. No linear or quadratic associations were identified for TEI (nor its factors) and fixating time for neutral ( $\mathrm{L}: \beta \mathrm{s} \leq .24, p s>.05$; $\mathrm{Q}: \beta \mathrm{s} \leq-.44, p s>.05$ ), socially threatening (L: $\beta \mathrm{s} \leq .-.09, p s>.62 ; \mathrm{Q}: \beta \mathrm{s} \leq-.04$, ps $>.89$ ) or physically threatening (L: $\beta \mathrm{s} \leq .09$, ps $>.05$; Q: $\beta \mathrm{s} \leq 2.17$, ps $>.05$ ) stimuli, regardless of time block. Linear, but not quadratic, associations between global TEI and fixation time for positive social scene approached significance in very early $(\mathrm{L}: \beta=.00, p=.07)$ and late $(\mathrm{L}: \beta=.00, p=.06)$ time blocks. Findings were significant for TEI factors. There was a positive linear relationship between the emotionality factor and attention to the positive social scene across all time blocks: very early $-\mathrm{F}(1,51)=4.83, p=.033$, adjusted $\mathrm{R}^{2}=.067, \beta=.29$; early $-\mathrm{F}(1,51)=$ 4.15, $p=.047$, adjusted $\mathrm{R}^{2}=.056, \beta=.27$, and late $-\mathrm{F}(1,51)=5.04, p=.029$, adjusted $\mathrm{R}^{2}=$ $.071, \beta=.30$ (see Figures 2-4). Additionally, the linear relationship between wellbeing and attention towards positive social stimuli approached significance in late time blocks $(\beta \leq .27$, $p=.051)$.

The scene of first fixation did not vary between those scoring high and low on TEI, $\left(\chi^{2}(1, N=54)=9.13, p=.701\right)$ : individuals, first, fixated on the social threat scene, regardless of TEI level.

Correlations between TEI and fixation times for each time block, across each task, are provided in Supplementary Material 1. 


\section{Discussion}

Deconstructing how EI relates to attentional processes remains a largely unexplored area of research. In the current study, using eye-tracking technology, and validated, ecologically salient emotional stimuli, we showed that TEI was associated with visual preferences for positive emotional stimuli.

\subsection{Trait EI and Visual Processing}

The present study identified positive relationships between TEI and positive emotional stimuli. More specifically, when confronted with crowds populated mostly with angry faces, individuals with higher TEI fixated longer on the happy faces, a pattern which also applied to the component factors of emotionality, self-control, and wellbeing. Furthermore, high TEI individuals showed a visual preference for positive social scenes (depicting relationships and social interaction) over social threat, physical threat, and neutral scenes, compared to their low TEI peers.

Those findings are consistent with the thesis that EI is associated with a bias for emotional over neutral information, an adaptive form of processing (Fiori, 2009). Although some laboratory outcomes contradict that claim (e.g. Matthews et al., 2015), empirical evidence is largely supportive: for example, high TEI individuals exhibit superior emotion recognition ability (Austin, 2004) and identify morphed facial expressions faster than low TEI scorers (Petrides \& Furnham, 2003). Current findings add to that literature and suggest the role of TEI extends to greater allocation of attentional resources towards positive emotional vs. neutral imagery, as well as emotionally valenced words vs. neutral words (Coffey, Berenbaum, \& Kerns, 2003; Mikolajczak et al., 2009).

However, the current findings are inconsistent with those from the only other eyetracking study to examine TEI. In Davis (in press), TEI emotionality (encapsulating traits of 
emotion perception, expression, and empathy) moderated visual attentional processes - high scorers showed bias towards sad faces relative to neutral faces; whereas we found emotionality effects in the opposite direction. Our key finding - the main effects of TEI (and constituent factors) on bias for positive emotional stimuli - also diverges from Davis’ findings., but could be explained by methodological differences between the studies, including: (1) the emotional stimuli used, (2) the stimuli presentation (two images versus four), (3), the viewing paradigm (time-limited, brief presentation of faces via a dot probe paradigm versus extended presentation of faces viewed passively). Although (4) the current study does not examine visual biases under stress, which is necessary in order to ascertain whether TEI is useful within high-stakes situations, effects identified were consistent across both stressful and non-stressful conditions in Davis (in press). Although (4) the current study does not examine visual biases under stress, which is necessary in order to ascertain whether TEI is useful within high-stakes situations, effects identified were consistent across both stressful and non-stressful conditions in Davis (in press). It could be argued that composite stimuli in the present study (i.e. crowds and scenes) are more ecologically valid, as emotional faces are rarely presented in isolation in everyday life. Moreover, our findings concerning positive emotion spanned two picture-viewing tasks with different emotional stimuli, suggesting a robust, generalised effect.

\subsection{Implications for the Adaptive Nature of Trait EI}

Empirical study of attentional bias generally shows that psychologically healthy and happy individuals show bias for positive emotion (Hakamata et al., 2010; Isaacowitz, Toner, Goren, \& Wilson, 2008; Weierich et al., 2008). We speculate that an inherent visual attentional preference for positive emotional material exhibited by high TEI individuals could form one pathway through which TEI facilitates improved psychological wellbeing. Specifically, individuals with higher TEI levels may be drawn more towards viewing 
emotional stimuli with positive, happy elements (over negative and neutral alternatives), which could, in turn, lead to better mental health. That effect could operate to maintain stability through a positive feedback loop: increased viewing of positive material improves mental health, with improved mental health subsequently increasing preferential viewing of positive material. A visual preference for positive emotion could also buffer the effects of situational stress by minimizing physiological reactivity, and promoting emotional recovery (Davis, in press). Considered along with findings of selective use of positive versus negative emotion regulation strategies by people higher in TEI (Szczygiel \& Mikolajczak, 2017), our findings help explain why emotionally intelligent individuals tend to show higher levels of wellbeing and happiness.

\subsection{Limitations}

Several limitations of the study require consideration. The present study did not include assessment of mood disorders or broadband personality covariates of TEI (Petrides, Pita, \& Kokkinaki, 2007; Weierich et al., 2008), meaning potential confounding influences cannot be confidently ruled out. In addition, a brief TEI measurement tool was selected due to time constraints. Although the TEIQue-SF reports excellent psychometric properties, the influence of the subscales should be interpreted with caution, since these comprise only 6-8 items. In the present study, the reliability coefficient for emotionality was only .67, and conclusions regarding its role should be reserved until consistent findings are demonstrated using the full-length TEIQue. The relatively small sample size $(n=54)$ should also be noted as a limitation in the present study. Although gender composition was unbalanced, effects remained significant when gender was controlled for (see Supplementary Material 2).

\subsection{Future Directions}


To evaluate the nature of TEI 'in action', further assessment of visual processing in challenging situations is needed. If TEI is truly adaptive, the patterns observed in the present study (preference for positive stimuli) should shift (preference for negative stimuli) for participants under acutely stressful conditions. AEI was not assessed in this study, but concurrently exploring the contributions of both TEI and AEI would allow for a more comprehensive examination of how EI influences visual attentional processing. In addition, it is unclear whether effects extend to other population groups, such as children/adolescents. Future studies should also include measures of personality, and mood disorders, to establish the incremental validity of TEI as a moderator of visual processing patterns.

\section{References}

Austin, E. J. (2004). An investigation of the relationship between trait emotional intelligence and emotional task performance. Personality and Individual Differences, 36(8), 18551864. https://doi.org/10.1016/j.paid.2003.07.006

Bar-Haim, Y., Lamy, D., Pergamin, L., Bakermans-Kranenburg, M. J., \& van IJzendoorn, M. H. (2007). Threat-related attentional bias in anxious and nonanxious individuals: a metaanalytic study. Psychological Bulletin, 133(1), 1-24. https://doi.org/10.1037/00332909.133.1.1

Brackett, M. A., Rivers, S. E., \& Salovey, P. (2011). Emotional Intelligence: Implications for Personal, Social, Academic, and Workplace Success. Social and Personality Psychology Compass, 5(1), 88-103. 10.1111/j.1751-9004.2010.00334.x

Buckner, J. D., Maner, J. K., \& Schmidt, N. B. (2010). Difficulty disengaging attention from social threat in social anxiety. Cognitive Therapy and Research, 34(1), 99-105. https://doi.org/10.1007/s10608-008-9205-y

Coffey, E., Berenbaum, H., \& Kerns, J. G. (2003). The dimensions of emotional intelligence, 
alexithymia, and mood awareness: Associations with personality and performance on an emotional stroop task. Cognition and Emotion, 17(4), 671-679.

https://doi.org/10.1080/02699930302304

Cooper, A., \& Petrides, K. V. (2010). A psychometric analysis of the Trait Emotional Intelligence Questionnaire-Short Form (TEIQue-SF) using item response theory. Journal of Personality Assessment, 92(5), 449-457.

https://doi.org/10.1080/00223891.2010.497426

Davis, S.K. (in press). Emotional intelligence and attentional bias for threat-related emotion. Scandanavian Journal of Psychology.

Davis, S. K., \& Nichols, R. (2016). Does Emotional Intelligence have a “Dark” Side? A Review of the Literature. Frontiers in Psychology, 7(August). https://doi.org/10.3389/fpsyg.2016.01316

Fiori, M. (2009). A new look at emotional intelligence: a dual-process framework. Personality and Social Psychology Review, 13(1), 21-44. https://doi.org/10.1177/1088868308326909

Goeleven, E., De Raedt, R., Leyman, L., \& Verschuere, B. (2008). The Karolinska Directed Emotional Faces: A validation study. Cognition \& Emotion, 22(6), 1094-1118. https://doi.org/10.1080/02699930701626582

Gutiérrez-Cobo, M. J., Cabello, R., \& Fernández-Berrocal, P. (2016). The Relationship between Emotional Intelligence and Cool and Hot Cognitive Processes: A Systematic Review. Frontiers in Behavioral Neuroscience, 10(May), 101. https://doi.org/10.3389/fnbeh.2016.00101

Hakamata, Y., Lissek, S., Bar-Haim, Y., Britton, J., Fox, N., Leibenluft, E., Ernst, M. \& Pine, 
D. (2010). Attentional Bias Modification Treatment: A meta-analysis towards the establishment of novel treatment for anxiety. Biological Psychiatry, 68(11), 982-990. https://doi.org/10.1007/s11103-011-9767-z.Plastid

Isaacowitz, D. M., Toner, K., Goren, D., \& Wilson, H. R. (2008). Looking while unhappy: mood-congruent gaze in young adults, positive gaze in older adults. Psychological Science, 19(9), 848-853. https://doi.org/10.1111/j.1467-9280.2008.02167.x.Looking

Jang, S., Kim, S., Kim, C., Lee, H., \& Choi, K. (2016). Attentional processing of emotional faces in schizophrenia: Evidence from eye tracking. Journal of Abnormal Psychology, 125(7), 894-906. https://doi.org/10.1037/abn0000198

Kress, L., \& Aue, T. (2017). The link between optimism bias and attention bias: A neurocognitive perspective. Neuroscience \& Biobehavioral Reviews, 80, 688-702. https://doi.org/10.1016/j.neubiorev.2017.07.016.

Lange, W. G., Heuer, K., Langner, O., Keijsers, G. P. J., Becker, E. S., \& Rinck, M. (2011). Face value: Eye movements and the evaluation of facial crowds in social anxiety. Journal of Behavior Therapy and Experimental Psychiatry, 42(3), 355-363. https://doi.org/10.1016/j.jbtep.2011.02.007

Martins, A., Ramalho, N., \& Morin, E. (2010). A comprehensive meta-analysis of the relationship between Emotional Intelligence and health. Personality and Individual Differences, 49(6), 554-564. https://doi.org/10.1016/j.paid.2010.05.029

Matthews, G., Perez-Gonzalez, J.-C., Fellner, a. N., Funke, G. J., Emo, a. K., Zeidner, M., \& Roberts, R. D. (2015). Individual Differences in Facial Emotion Processing: Trait Emotional Intelligence, Cognitive Ability, or Transient Stress? Journal of Psychoeducational Assessment, 33(1), 68-82. https://doi.org/10.1177/0734282914550386 
Mayer, J. D., Roberts, R. D., \& Barsade, S. G. (2008). Human Abilities: Emotional Intelligence. Annual Review of Psychology, 59(1), 507-536. https://doi.org/10.1146/annurev.psych.59.103006.093646

Mikolajczak, M., Roy, E., Verstrynge, V., \& Luminet, O. (2009). An exploration of the moderating effect of trait emotional intelligence on memory and attention in neutral and stressful conditions. British Journal of Psychology, 100 (4), 699-715. https://doi.org/10.1348/000712608X395522

Nelis, D., Quoidbach, J., Mikolajczak, M., \& Hansenne, M. (2009). Increasing emotional intelligence: (How) is it possible? Personality and Individual Differences, 47(1), 36-41. https://doi.org/10.1016/j.paid.2009.01.046

Petrides, K. V. (2009). Psychometric properties of the Trait Emotional Intelligence Questionnaire. In C. Stough, D. H. Saklofske, and J. D. Parker, Advances in the assessment of emotional intelligence. New York: Springer.

Petrides, K. V., \& Furnham, A. (2003). Trait Emotional Intelligence: Behavioural Validation in Two Studies of Emotion Recognition and Reactivity to Mood Induction. European Journal of Personality, 17(1), 39-57. https://doi.org/10.1002/per.466

Petrides, K. V., Mikolajczak, M., Mavroveli, S., Sánchez-Ruiz, M-J., Furnham, A., \& PérezGonzález, J-C. (2016). Developments in trait emotional intelligence research. Emotion Review, 8, 335-341. https://doi.org/10.1177/1754073916650493

Petrides, K.V., Pita, R., \& Kokkinaki, F. (2007). The location of trait emotional intelligence in personality factor space. British Journal of Psychology, 98, 273-289.

DOI:10.1348/000712606X120618

Pinkham, A. E., Griffin, M., Baron, R., Sasson, N. J., \& Gur, R. C. (2010). The face in the 
crowd effect: Anger superiority when using real faces and multiple identities. Emotion, 10(1), 141-146. https://doi.org/10.1037/a0017387

Qualter, P., Whiteley, H.E., \& Pope, D.J. (2007). Supporting the development of emotional intelligence competencies to ease the transition from primary to high school.

Educational Psychology in Practice, 23(1), 79-95.

https://doi.org/10.1080/02667360601154584

Weierich, M. R., Treat, T. a., \& Hollingworth, A. (2008). Theories and measurement of visual attentional processing in anxiety. Cognition \& Emotion (Vol. 22).

https://doi.org/10.1080/02699930701597601

Yiend, J. (2010). The effects of emotion on attention: A review of attentional processing of emotional information. Cognition and Emotion, 24(May 2013), 37-41. https://doi.org/10.1080/02699930903205698

Figure 1. Percentage fixation time on happy faces in a crowd of 6 happy and 10 angry faces in Task 2. 


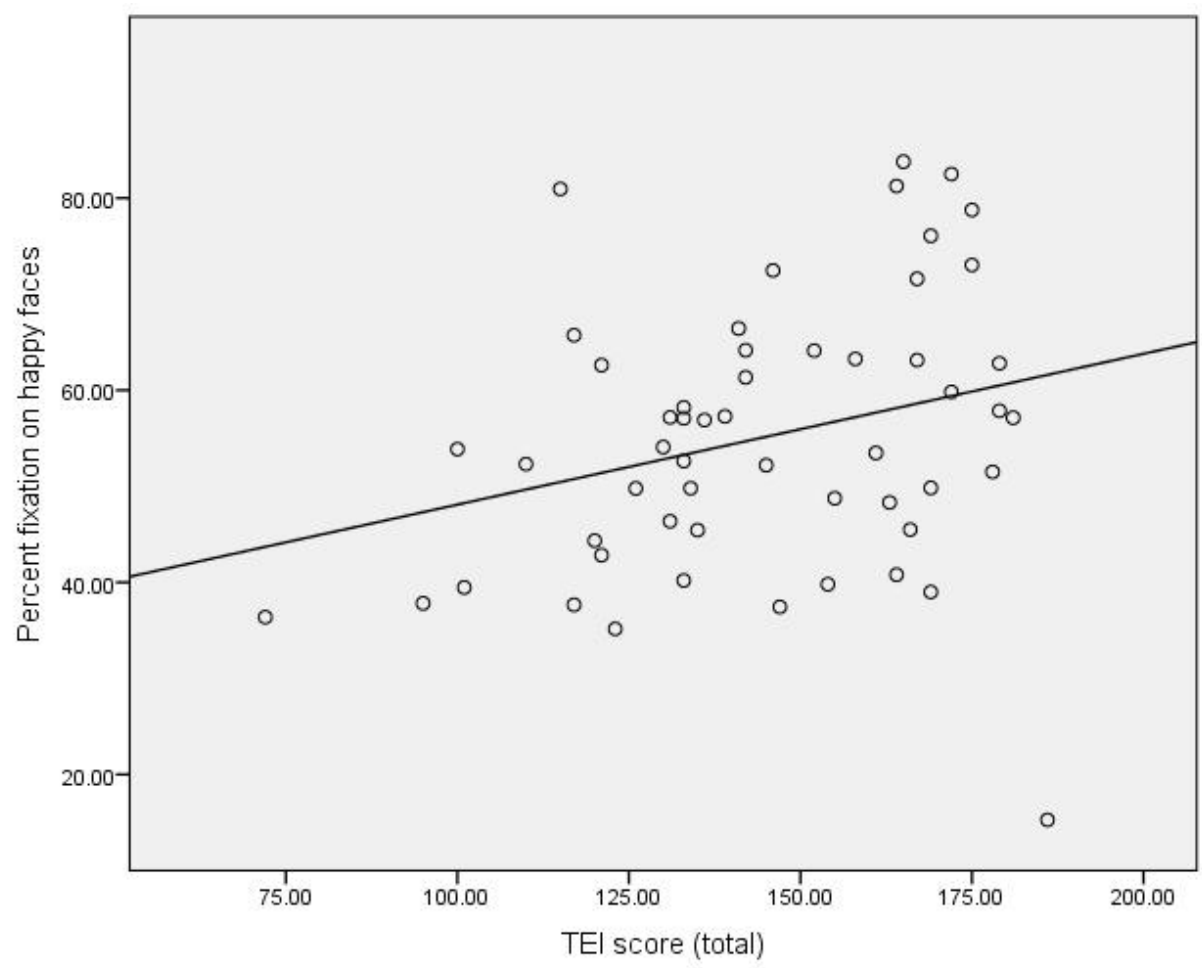

Figure 2. Percentage fixation time on positive social scenes for very early time blocks (0500ms) in Task 3. 


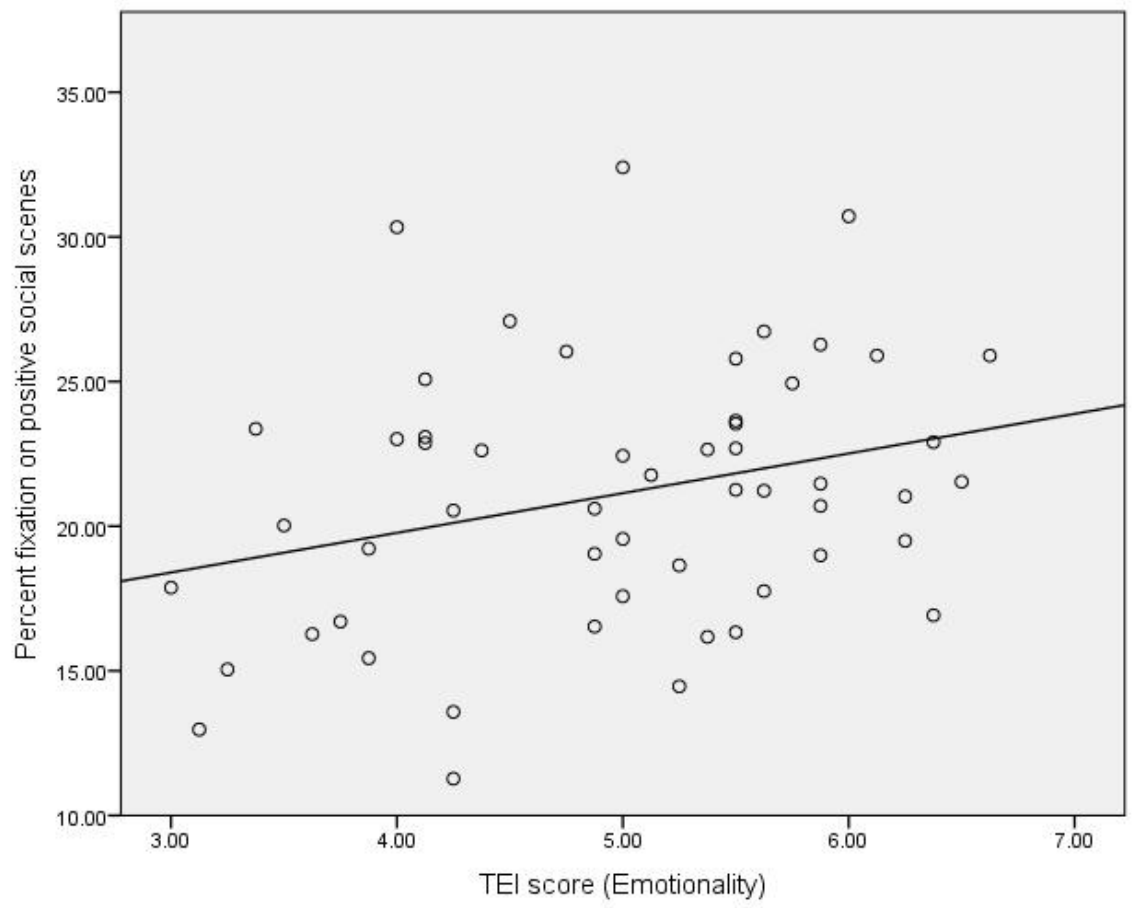

Figure 3. Percentage fixation time on positive social scenes for early time blocks (5004000ms) in Task 3.

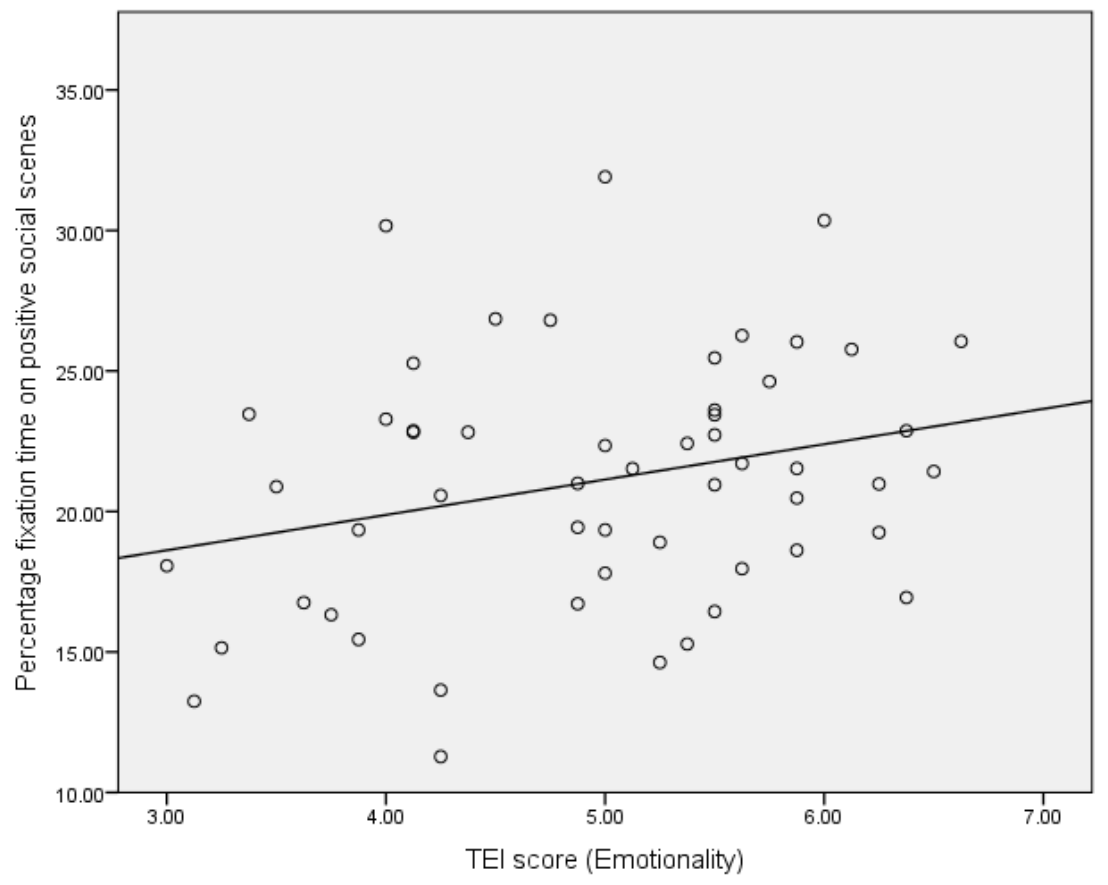

Figure 4. Percentage fixation time on positive social scenes for late time blocks (4000$8000 \mathrm{~ms})$ in Task 3. 


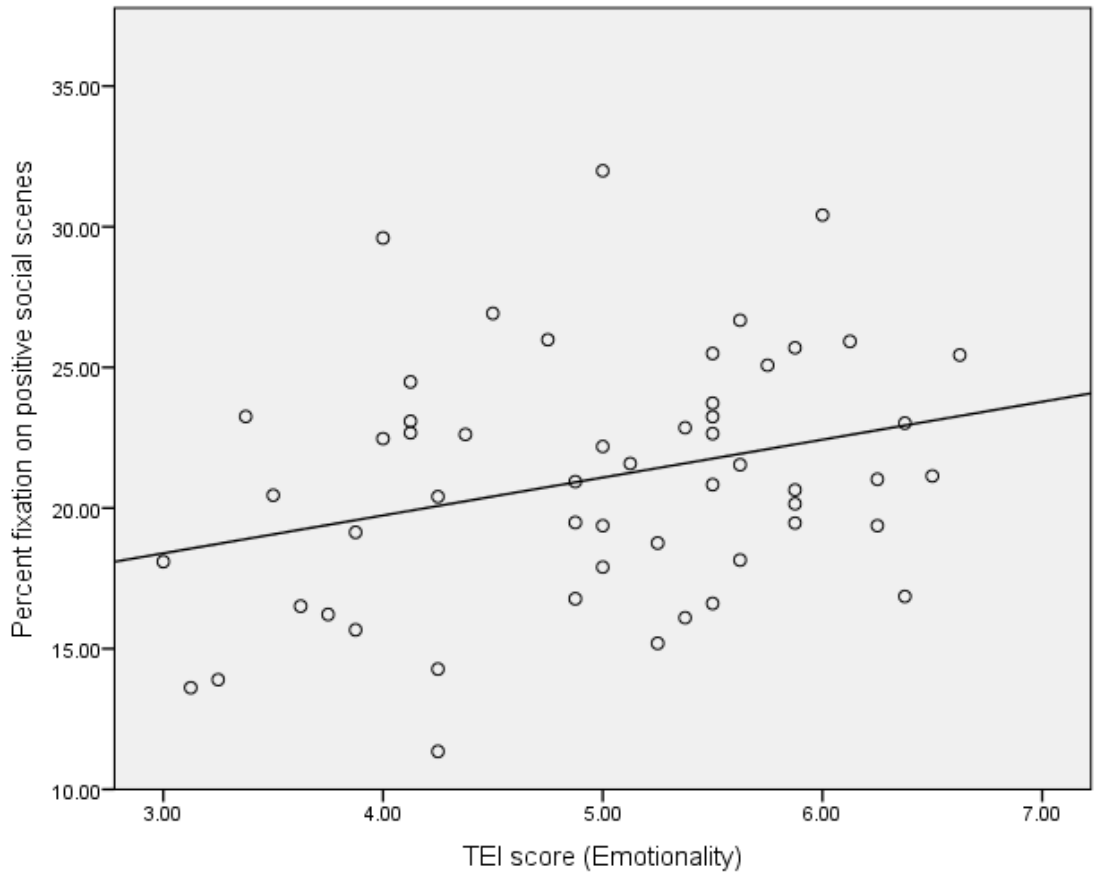

